\title{
Strict density topology on the plane. Measure case
}

\author{
Małgorzata Filipczak • Władysław Wilczyński
}

Received: 16 March 2010 / Accepted: 29 November 2010 / Published online: 8 June 2011

(C) The Author(s) 2011. This article is published with open access at Springerlink.com

Abstract We present a density-type topology on the plane generated by the strict convergence of double sequences. The point of restricted density of a measurable set $A \subset \mathbb{R} \times \mathbb{R}$ is defined using a convergence in a restricted sense of a sequence of a form

$$
s_{n, m}=\frac{\lambda_{2}\left(A \cap\left(\left[-\frac{1}{n} ; \frac{1}{n}\right] \times\left[-\frac{1}{m} ; \frac{1}{m}\right]\right)\right)}{4 \frac{1}{n} \frac{1}{m}} .
$$

The definition of a strict density point is a natural modification of the previous notion. We describe operators and topologies generated by them.

Keywords Strong density on the plane - Convergence in the restricted sense - Density-type topologies

Mathematics Subject Classification (2000) Primary 54A10 - Secondary 28A05 · 28A15

We shall start from classical definitions of density on the real line and strong density on the plane. Let $\mathcal{L}_{1}$ be a $\sigma$-algebra of Lebesgue measurable subsets of $\mathbb{R}$ and $\lambda_{1}$ - a Lebesgue measure on the real line. A point $x_{0}$ is called a density point of a set $E \in \mathcal{L}_{1}$ if

$$
\lim _{h \rightarrow 0^{+}} \frac{\lambda_{1}\left(E \cap\left[x_{0}-h ; x_{0}+h\right]\right)}{2 h}=1 .
$$

By $\Phi(E)$ we denote a set of all density points of $E$.

\footnotetext{
M. Filipczak $(\bowtie) \cdot$ W. Wilczyński

Faculty of Mathematics and Computer Sciences, Łódź University, ul. S. Banacha 22, 90-238 Łódź,

Poland

e-mail: malfil@math.uni.lodz.pl

W. Wilczyński

e-mail:wwil@uni.lodz.pl
} 
Let $\mathcal{L}_{2}$ be a $\sigma$-algebra of Lebesgue measurable subsets of $\mathbb{R}^{2}$ and $\lambda_{2}$ - a two-dimensional Lebesgue measure. We say that $\left(x_{0}, y_{0}\right) \in \mathbb{R}^{2}$ is a a point of strong density of $A \in \mathcal{L}_{2}$ if

$$
\lim _{\substack{h \rightarrow 0^{+} \\ k \rightarrow 0^{+}}} \frac{\lambda_{2}\left(A \cap\left(\left[x_{0}-h ; x_{0}+h\right] \times\left[y_{0}-k ; y_{0}+k\right]\right)\right)}{4 h k}=1 .
$$

Note, that $(0,0)$ is a point of strong density of $A$ if and only if a double sequence $\left\{s_{n, m}\right\}_{n, m \in N}$ defined by the formula

$$
s_{n, m}=\frac{\lambda_{2}\left(A \cap\left(\left[-\frac{1}{n} ; \frac{1}{n}\right] \times\left[-\frac{1}{m} ; \frac{1}{m}\right]\right)\right)}{4 \frac{1}{n} \frac{1}{m}}
$$

converges to 1 . Clearly, if $(0,0)$ is a point of strong density of $A$ then $\lim _{\substack{n \rightarrow \infty \\ m \rightarrow \infty}} s_{n, m}=1$. Moreover, for any $h, k \in(0,1)$ there are $n, m \in \mathbb{N}$ such that $\frac{1}{n+1}<h \leq \frac{1}{n}$ and $\frac{1}{m+1}<k \leq \frac{1}{m}$. Hence

$$
\begin{aligned}
1 & \geq \frac{\lambda_{2}(A \cap([h ; h] \times[-k ; k]))}{4 h k} \geq \frac{\lambda_{2}\left(A \cap\left(\left[-\frac{1}{n+1} ; \frac{1}{n+1}\right] \times\left[-\frac{1}{m+1} ; \frac{1}{m+1}\right]\right)\right)}{4 \frac{1}{n} \frac{1}{m}} \\
& =\frac{\lambda_{2}\left(A \cap\left(\left[-\frac{1}{n+1} ; \frac{1}{n+1}\right] \times\left[-\frac{1}{m+1} ; \frac{1}{m+1}\right]\right)\right)}{4 \frac{1}{n+1} \frac{1}{m+1}} \cdot \frac{n+1}{n} \cdot \frac{m+1}{m}
\end{aligned}
$$

and if $\lim _{\substack{n \rightarrow \infty \\ m \rightarrow \infty}} s_{n, m}=1$, then $\lim _{\substack{h \rightarrow 0^{+} \\ k \rightarrow 0^{+}}} \frac{\lambda_{2}(A \cap([h ; h] \times[-k ; k]))}{4 h k}=1$.

In [5], p. 18 one can find the following definition: a double sequence $\left\{s_{n, m}\right\}_{n, m \in N}$ of real numbers converges in the restricted sense to $s$ if for each $\varepsilon>0$ there exists $n_{0} \in \mathbb{N}$ such that $\left|s_{m, n}-s\right|<\varepsilon$ whenever $n+m>n_{0}$. It is not difficult to observe that the above condition is equivalent to the conjunction of the following three:

(a) $\lim _{n \rightarrow \infty} s_{n, m}=s$ for each $m \in \mathbb{N}$;

(b) $\lim _{m \rightarrow \infty} s_{n, m}=s$ for each $n \in \mathbb{N}$;

(c) $\lim _{\substack{n \rightarrow \infty \\ m \rightarrow \infty}} s_{n, m}=s$.

We shall say that $(0,0)$ is a point of restricted density of $A \in \mathcal{L}_{2}$ if a double sequence $\left\{s_{n, m}\right\}_{n, m \in N}$ defined by the formula

$$
s_{n, m}=\frac{\lambda_{2}\left(A \cap\left(\left[-\frac{1}{n} ; \frac{1}{n}\right] \times\left[-\frac{1}{m} ; \frac{1}{m}\right]\right)\right)}{4 \frac{1}{n} \frac{1}{m}}
$$

converges to 1 in the restricted sense. If $(0,0)$ is a point of restricted density of $\mathbb{R}^{2} \backslash A$ then we shall say that $(0,0)$ is a point of restricted dispersion of $A$. It means that $(0,0)$ is a point of restricted density of a measurable set $A$ if and only if the conjunction of the following three conditions hold:

(a1) $\lim _{n \rightarrow \infty} \frac{\lambda_{2}\left(A \cap\left(\left[-\frac{1}{n} ; \frac{1}{n}\right] \times\left[-\frac{1}{m} ; \frac{1}{m}\right]\right)\right)}{4 \frac{1}{n} \frac{1}{m}}=1$ for each $m \in \mathbb{N}$;

(b1) $\lim _{m \rightarrow \infty} \frac{\lambda_{2}\left(A \cap\left(\left[-\frac{1}{n} ; \frac{1}{n}\right] \times\left[-\frac{1}{m} ; \frac{1}{m}\right]\right)\right)}{4 \frac{1}{n} \frac{1}{m}}=1$ for each $n \in \mathbb{N}$;

(c1) $\lim _{\substack{n \rightarrow \infty \\ m \rightarrow \infty}} \frac{\lambda_{2}\left(A \cap\left(\left[-\frac{1}{n} ; \frac{1}{n}\right] \times\left[-\frac{1}{m} ; \frac{1}{m}\right]\right)\right)}{4 \frac{1}{n} \frac{1}{m}}=1$. 
We have shown that $(\mathrm{c} 1)$ is equivalent to the fact that $(0,0)$ is a point of strong density of $A$. In the same way we can check that (a1) is equivalent to the condition:

$$
\lim _{h \rightarrow 0^{+}} \frac{\lambda_{2}(A \cap([-h ; h] \times[-k ; k]))}{4 h k}=1 \quad \text { for each } k \in(0 ; 1)
$$

and the analogous property is true for (b1). Moreover, because of the specific form of our sequence, the condition (a1) holds if and only if

$$
\lim _{n \rightarrow \infty} \frac{\lambda_{2}\left(A \cap\left(\left[-\frac{1}{n} ; \frac{1}{n}\right] \times[-1 ; 1]\right)\right)}{4 \frac{1}{n}}=1,
$$

i.e. $s_{n, 1} \underset{n \rightarrow \infty}{\rightarrow} 1$.

Summarising, we obtain the following

Proposition 1 The point $(0,0)$ is a point of restricted density of a measurable set $A$ if and only if the conjunction of the following three conditions hold:

(a2) $\lim _{n \rightarrow \infty} \frac{\lambda_{2}\left(A \cap\left(\left[-\frac{1}{n} ; \frac{1}{n}\right] \times[-1 ; 1]\right)\right)}{4 \frac{1}{n}}=1$;

(b2) $\lim _{m \rightarrow \infty} \frac{\lambda_{2}\left(A \cap\left([-1 ; 1] \times\left[-\frac{1}{m} ; \frac{1}{m}\right]\right)\right)}{4 \frac{1}{m}}=1$;

(c2) $(0,0)$ is a point of strong density of $A$.

A point $\left(x_{0}, y_{0}\right) \in \mathbb{R}^{2}$ will be called a a point of restricted density (dispersion) of a set $A$ if $(0,0)$ is a point of restricted density (dispersion) of a set $A-\left(x_{0}, y_{0}\right)=\left\{\left(x-x_{0}, y-y_{0}\right)\right.$ : $(x, y) \in A\}$. We will denote by $\Phi_{r}(A)$ the set of all restricted density points of $A$. It is easy to check that if $\lambda_{2}(P)=0$ then $\Phi_{r}\left(\mathbb{R}^{2} \backslash P\right)=\mathbb{R}^{2}$ and $\Phi_{r}((0 ; 1) \times(0 ; 1))=\varnothing$. Note, that every point of the set $(0 ; 1) \times(0 ; 1)$ is a point of its strong density. Moreover, if $x \in \mathbb{R}$ is a density point of a measurable set $E \subset \mathbb{R}$, then $(x, y)$ is a strong density point of $E \times \mathbb{R}$. The notion of restricted density is much stronger.

Theorem 1 If $b-a \leq 1$ and $\lambda_{1}((a ; b) \backslash E)>0$ then no point $(x, y) \in(E \cap(a ; b)) \times \mathbb{R}$ is a restricted density point of the set $A=E \times \mathbb{R}$.

Proof Observe that if $x_{0} \in E \cap(a ; b)$ then

$$
\frac{\lambda_{1}\left(\left[x_{0}-1 ; x_{0}+1\right] \cap E\right)}{2}<1 .
$$

Therefore, for any $y \in \mathbb{R}$,

$$
\lim _{m \rightarrow \infty} \frac{\lambda_{2}\left(\left(\left[x_{0}-1 ; x_{0}+1\right] \times\left[y-\frac{1}{m} ; y+\frac{1}{m}\right]\right) \cap A\right)}{4 \frac{1}{m}}=\frac{\lambda_{1}\left(\left[x_{0}-1 ; x_{0}+1\right] \cap E\right)}{2}<1
$$

and $\left(x_{0}, y\right)$ is not a restricted density point of $A$.

Example 1 There exists a measurable set $A \subset \mathbb{R}^{2}$ such that $(0,0)$ is a point of restricted dispersion of $A$ and for any $h>0$

$$
\lambda_{2}(A \cap([-h ; h] \times[-h ; h]))>0 .
$$


Let

$$
A=\left\{(x, y): 0 \leq x, 0 \leq y, x \leq y \leq x+x^{2}\right\} .
$$

Fix $h$ and $k$ from $(0 ; 1)$ and denote by $l$ the minimum of $h$ and $k$. Then

$$
A \cap([-h ; h] \times[-k ; k]) \subset A \cap\left([0 ; l] \times\left[0 ; l+l^{2}\right]\right)
$$

and

$$
\frac{\lambda_{2}(A \cap([-h ; h] \times[-k ; k]))}{4 h k}<\frac{\frac{1}{2} l \cdot l^{2}}{4 l^{2}}=\frac{1}{8} l .
$$

Therefore $\lim _{\substack{h \rightarrow 0^{+} \\ k \rightarrow 0^{+}}} \frac{\lambda_{2}(A \cap([h ; h] \times[-k ; k]))}{4 h k}=0$. Moreover, for any fixed $h \in(0 ; 1)$ and $k<h$,

$$
\frac{\lambda_{2}(A \cap([-h ; h] \times[-k ; k]))}{4 h k}<\frac{1}{8} k \underset{k \rightarrow 0^{+}}{\rightarrow} 0
$$

and—analogously—for any $k \in(0 ; 1)$

$$
\lim _{h \rightarrow 0^{+}} \frac{\lambda_{2}(A \cap([-h ; h] \times[-k ; k]))}{4 h k}=0 .
$$

Observe that $(0,0)$ is not a point of restricted dispersion of a set defined by the formula

$$
B=\left\{(x, y): 0 \leq x, 0 \leq y, 0 \leq y \leq x^{2}\right\} .
$$

Indeed, for any $m \geq 2$

$$
\frac{\lambda_{2}\left(B \cap\left([-1 ; 1] \times\left[-\frac{1}{m} ; \frac{1}{m}\right]\right)\right)}{4 \frac{1}{m}} \geq \frac{\left(1-\frac{1}{\sqrt{m}}\right) \cdot \frac{1}{m}}{4 \frac{1}{m}}=\frac{1}{4}\left(1-\frac{1}{\sqrt{m}}\right) .
$$

The sequence $\left\{\frac{1}{4}\left(1-\frac{1}{\sqrt{m}}\right)\right\}_{m \in N}$ tends to $\frac{1}{4}$, so $(0,0)$ is not a point of restricted dispersion of $B$.

Theorem 2 Suppose that $A \in \mathcal{L}_{2}$. If $A \subset \Phi_{r}(A)$ then $A=\varnothing$ or $\lambda_{2}\left(\mathbb{R}^{2} \backslash A\right)=0$.

Proof Let $A$ be a nonempty set and $A \subset \Phi_{r}(A)$. Fix a point $\left(x_{a}, y_{a}\right) \in A$. We shall prove that

$$
\lambda_{2}\left(\left(\left[x_{a}-1 ; x_{a}+1\right] \times\left[y_{a}-1 ; y_{a}+1\right]\right) \backslash A\right)=0 .
$$

Denote by $S$ the square $\left[x_{a}-1 ; x_{a}+1\right] \times\left[y_{a}-1 ; y_{a}+1\right]$ and suppose that the set $B=S \backslash A$ has a positive measure. For the simplicity, assume that

$$
\lambda_{2}\left(B \cap\left(\left[x_{a} ; x_{a}+1\right] \times\left[y_{a} ; y_{a}+1\right]\right)\right)>0 .
$$

There exist numbers $b, c, d, e$ such that $x_{a}<b<c<x_{a}+1, y_{a}<d<e<y_{a}+1$ and the set $C=B \cap([b ; c] \times[d ; e])$ has positive measure. Denote by $C^{y}$ the set $\{x:(x, y) \in C\}$. From the Fubini Theorem it follows that the function $\Psi(y)=\lambda_{1}\left(C^{y}\right)$, defined almost everywhere on $\operatorname{pr}_{X}(C)$, is measurable and

$$
\lambda_{1}(\{y: \Psi(y)>0\})=\lambda_{1}\left(\bigcup_{n=1}^{\infty}\left\{y: \Psi(y)>\frac{1}{n}\right\}\right)>0 .
$$


Hence, there exists $n_{0}>0$ such that a set

$$
D=\left\{y: \Psi(y)>\frac{1}{n_{0}}\right\}
$$

has positive measure. From Lebesgue Density Theorem ([3], Theorem 3.20) we know that $\lambda_{1}(D \cap \Phi(D))>0$. We shall show that for any $x_{1} \in(c-1 ; b)$ and $y_{1} \in D \cap \Phi(D)$, the point $\left(x_{1}, y_{1}\right)$ is not a point of restricted density of $A$. Since $y_{1} \in \Phi(D)$, then there exists $m_{0} \in \mathbb{N}$ such that for any $m>m_{0}$,

$$
\lambda_{1}\left(D \cap\left[y_{1}-\frac{1}{m} ; y_{1}+\frac{1}{m}\right]\right)>\frac{1}{m} .
$$

Therefore,

$$
\begin{gathered}
\frac{\lambda_{2}\left(\left(\mathbb{R}^{2} \backslash A\right) \cap\left(\left[x_{1}-1 ; x_{1}+1\right] \times\left[y_{1}-\frac{1}{m} ; y_{1}+\frac{1}{m}\right]\right)\right)}{4 \frac{1}{m}} \\
\geq \frac{\lambda_{2}\left(C \cap\left(\left[x_{1}-1 ; x_{1}+1\right] \times\left[y_{1}-\frac{1}{m} ; y_{1}+\frac{1}{m}\right]\right)\right)}{4 \frac{1}{m}} \\
\geq \frac{\frac{1}{\eta_{0}} \cdot \lambda_{1}\left(D \cap\left[y_{1}-\frac{1}{m} ; y_{1}+\frac{1}{m}\right]\right)}{\frac{4}{m}}>\frac{1}{4 \eta_{0}} .
\end{gathered}
$$

It follows that $\left(x_{1}, y_{1}\right)$ is not a point of restricted density of $A$.

From the assumption $A \subset \Phi_{r}(A)$, we obtain that $\left(x_{1}, y_{1}\right) \notin A$. Hence,

$$
(c-1 ; b) \times(D \cap \Phi(D)) \subset \mathbb{R}^{2} \backslash A .
$$

Let $k_{0}$ be such a positive integer that $x_{a}-\frac{1}{k_{0}}>c-1$ and $x_{a}+\frac{1}{k_{0}}<b$. For any $k \geq k_{0}$

$$
\frac{\lambda_{2}\left(\left(\left[x_{a}-\frac{1}{k} ; x_{a}+\frac{1}{k}\right] \times\left[y_{a}-1 ; y_{a}+1\right]\right) \backslash A\right)}{\frac{4}{k}} \geq \frac{1}{2} \lambda_{1}(D \cap \Phi(D)) .
$$

It follows that $\left(x_{a}, y_{a}\right)$ is not a point of restricted density of $A$ (because the condition (a2) fails). However $\left(x_{a}, y_{a}\right) \in A \subset \Phi_{r}(A)$. This contradiction proves that $\lambda_{2}\left((S \backslash A) \cap\left(\left[x_{a} ; x_{a}+\right.\right.\right.$ $\left.\left.1] \times\left[y_{a} ; y_{a}+1\right]\right)\right)=0$. The proof for other quarters of the square $S$ are analogous. We have proved that $\lambda_{2}\left(\left(\left[x_{a}-1 ; x_{a}+1\right] \times\left[y_{a}-1 ; y_{a}+1\right]\right) \backslash A\right)=0$ for any $\left(x_{a}, y_{a}\right) \in A$.

Now, fix a point $(\bar{x}, \bar{y}) \in A$. We know that

$$
\lambda_{2}(([\bar{x}-1 ; \bar{x}+1] \times[\bar{y}-1 ; \bar{y}+1]) \backslash A)=0 .
$$

Consider a rectangle $R=(\bar{x}-1 ; \bar{x}+1) \times[\bar{y}+1 ; \bar{y}+2)$. If there is a rectangle $[b ; c] \times$ $[d ; e] \subset R$ with $\lambda_{2}(([b ; c] \times[d ; e]) \backslash A)>0$ and $c-b<1$ then there exists a point

$$
\left(x_{a}, y_{a}\right) \in A \cap([b ; c] \times[e-1 ; \bar{y}+1]) .
$$

A square $\left[x_{a}-1 ; x_{a}+1\right] \times\left[y_{a}-1 ; y_{a}+1\right]$ contains $[b ; c] \times[d ; e]$, so

$$
\lambda_{2}\left(\left(\left[x_{a}-1 ; x_{a}+1\right] \times\left[y_{a}-1 ; y_{a}+1\right]\right) \backslash A\right)>0
$$


which gives a contradiction. Hence

$$
\lambda_{2}(R \backslash A)=0 .
$$

Proceeding by induction, we obtain that

$$
\lambda_{2}(([\bar{x}-1 ; \bar{x}+1] \times \mathbb{R}) \backslash A)=0
$$

and, in analogous way, that

$$
\lambda_{2}\left(\mathbb{R}^{2} \backslash A\right)=0 .
$$

In the proof of the above theorem we use only conditions (a2) and (b2) - omitting (c2). In fact, we have proved that if for any $\left(x_{a}, y_{a}\right) \in A$ the set $A-\left(x_{a}, y_{a}\right)$ satisfies (a2) and (b2), then $A=\varnothing$ or $\lambda_{2}\left(\mathbb{R}^{2} \backslash A\right)=0$. There is a natural question if there exists such measurable set $A \subset \mathbb{R}^{2}$ that (a2) and (b2) are fullfiled and $(0,0)$ is not a point of strong density of $A$.

Example 2 Let $B=\left\{(x, y): x^{2} \leq|y| \leq \sqrt{|x|}\right\}$ and $A=\mathbb{R}^{2} \backslash B$. For any $n \in N$

$$
\frac{\lambda_{2}\left(A \cap\left(\left[-\frac{1}{n} ; \frac{1}{n}\right] \times[-1 ; 1]\right)\right)}{4 \frac{1}{n}} \geq 1-\frac{1}{n^{2}}
$$

and (a2) is fullfiled. Similarly $\lim _{m \rightarrow \infty} \frac{\lambda_{2}\left(A \cap\left([-1 ; 1] \times\left[-\frac{1}{m} ; \frac{1}{m}\right]\right)\right)}{4 \frac{1}{m}}=1$. Moreover, for any $h>0$

$$
\frac{\lambda_{2}(A \cap([-h ; h] \times[-h ; h]))}{4 h^{2}}=\frac{8 \int_{0}^{h} x^{2} d x}{4 h^{2}}=\frac{2}{3} h,
$$

so $(0,0)$ is not a strong density point of $A$. (In fact $(0,0)$ is an ordinary dispersion point of $A$.)

Denote by $A \triangle B$ the symmetric difference of sets $A$ and $B$.

Proposition 2 The operator $\Phi_{r}: \mathcal{L}_{2} \rightarrow 2^{\mathbb{R}^{2}}$ has the following properties:

(1) for each $A, B \in \mathcal{L}_{2}$, if $\lambda_{2}(A \triangle B)=0$, then $\Phi_{r}(A)=\Phi_{r}(B)$;

(2) $\Phi_{r}(\varnothing)=\varnothing$ and $\Phi_{r}\left(\mathbb{R}^{2}\right)=\mathbb{R}^{2}$;

(3) for each $A, B \in \mathcal{L}_{2}, \Phi_{r}(A \cap B)=\Phi_{r}(A) \cap \Phi_{r}(B)$.

Proof The conditions (1) and (2) follow immediately from the definition. To prove (3) it is enough to check that if $(0,0)$ is a point of restricted dispersion of a sets $A$ and $B$, then it is a point of restricted dispersion of $A \cup B$.

Similar properties have the operator $\Phi$ of density on the real line and the operator $\Phi_{s}-$ of strong density, and a few other "density type" operators. Using such operator we construct a "density type" topology.

Theorem 3 A family $\mathcal{T}_{r}=\left\{A \in \mathcal{L}_{2}: A \subset \Phi_{r}(A)\right\}$ is a topology incomparable with the natural topology on the plane. A topological space $\left(\mathbb{R}^{2}, \mathcal{T}_{r}\right)$ is not a Hausdorff space. 
Proof Straightforward from Theorem 2 we obtain

$$
\mathcal{T}_{r}=\left\{A \subset \mathbb{R}^{2}: A=\varnothing \text { or } A=\mathbb{R}^{2} \backslash P, \lambda_{2}(P)=0\right\} .
$$

It is clear that this family forms a topology. It is $T_{1}$ but not $T_{2}$.

Remark 1 We can define an operator weaker than $\Phi_{r}$ generating the same topology. Let for each $A \in \mathcal{L}_{2}$ a set $\Phi_{r-}(A)$ consists of all points $(x, y)$ such that

$$
\lim _{n \rightarrow \infty} \frac{\lambda_{2}\left(A \cap\left(\left[x-\frac{1}{n} ; x+\frac{1}{n}\right] \times[y-1 ; y+1]\right)\right)}{\frac{4}{n}}=1
$$

and

$$
\lim _{m \rightarrow \infty} \frac{\lambda_{2}\left(A \cap\left([x-1 ; x+1] \times\left[y-\frac{1}{m} ; y+\frac{1}{m}\right]\right)\right)}{\frac{4}{m}}=1 .
$$

In other words, $(0,0) \in \Phi_{r-}(A)$ if and only if conditions (a2) and (b2) from Proposition 1 are fulfilled. From Example 2 it follows that an operator $\Phi_{r-}$ is different than $\Phi_{r}$. It is not difficult to check that $\Phi_{r-}$ satisfies the conditions (1)-(3) from Proposition 2. From the proof of Theorem 2 it appears that

$$
\begin{aligned}
\mathcal{T}_{r-} & =\left\{A \in \mathcal{L}_{2}: A \subset \Phi_{r-}(A)\right\} \\
& =\left\{A \subset \mathbb{R}^{2}: A=\varnothing \text { or } A=\mathbb{R}^{2} \backslash P, \lambda_{2}(P)=0\right\}=\mathcal{T}_{r} .
\end{aligned}
$$

Remark 2 Let $k, l$ be fixed natural numbers. Define for each $A \in \mathcal{L}_{2}$ a set $\Phi_{r k l}(A)$ consists of all points $(x, y)$ such that

$$
\begin{aligned}
& \lim _{n \rightarrow \infty} \frac{\lambda_{2}\left(A \cap\left(\left[x-\frac{1}{n} ; x+\frac{1}{n}\right] \times\left[y-\frac{1}{k} ; y+\frac{1}{k}\right]\right)\right)}{\frac{4}{n k}}=1 ; \\
& \lim _{m \rightarrow \infty} \frac{\lambda_{2}\left(A \cap\left(\left[x-\frac{1}{l} ; x+\frac{1}{l}\right] \times\left[y-\frac{1}{m} ; y+\frac{1}{m}\right]\right)\right)}{\frac{4}{m l}}=1 .
\end{aligned}
$$

and $(x, y)$ is a strong density point of $A$. For different pairs $k_{1}, l_{1}$ and $k_{2}, l_{2}$ the operators $\Phi_{r k_{1} l_{1}}$ and $\Phi_{r k_{2} l_{2}}$ are also different, but for any $k, l$ we can repeat the proof of Theorem 2 and again we obtain

$$
\begin{aligned}
\mathcal{T}_{r k l} & =\left\{A \in \mathcal{L}_{2}: A \subset \Phi_{r k l}(A)\right\} \\
& =\left\{A \subset \mathbb{R}^{2}: A=\varnothing \text { or } A=\mathbb{R}^{2} \backslash P, \lambda_{2}(P)=0\right\}=\mathcal{T}_{r} .
\end{aligned}
$$

Remark 3 In [2] it is proved that we obtain the same topology starting from a notion of area density. A point $(x, y) \in \mathbb{R}^{2}$ is called an area density point of a set $A \in \mathcal{L}_{2}$ if for each $\varepsilon>0$ there exists $\delta>0$ such that

$$
\frac{\lambda_{2}(A \cap([x-h ; x+h] \times[y-k ; y+k]))}{4 h k}>1-\varepsilon
$$

for each positive $h$ and $k$, if only $4 h k<\delta$. If we denote by $\Phi_{a}(A)$ the set of all area points of $A$ then 


$$
\begin{aligned}
\mathcal{T}_{a} & =\left\{A \in \mathcal{L}_{2}: A \subset \Phi_{a}(A)\right\} \\
& =\left\{A \subset \mathbb{R}^{2}: A=\varnothing \text { or } A=\mathbb{R}^{2} \backslash P, \lambda_{2}(P)=0\right\}=\mathcal{T}_{r}
\end{aligned}
$$

(compare [2, Theorem 4]).

From Theorem 3 and Remarks 1, 2 and 3 it appears that we obtain the topology $\mathcal{T}_{r}$ whenever we consider density defined by "long" intervals. The topology $\mathcal{T}_{r}$ is interesting but rather "poor" and incomparable with the natural topology on the plane. Now, we shall define topology connected with "long" - in some sense - intervals, but stronger then the natural topology.

Let $A \in \mathcal{L}_{2},(x, y) \in \mathbb{R}^{2}$ and

$$
s_{n, m}(x, y)=\frac{\lambda_{2}\left(A \cap\left(\left[x-\frac{1}{n} ; x+\frac{1}{n}\right] \times\left[y-\frac{1}{m} ; y+\frac{1}{m}\right]\right)\right)}{4 \cdot \frac{1}{n} \cdot \frac{1}{m}} .
$$

We say that $(x, y)$ is a strict density point of $A$ if there exist $n_{0}, m_{0} \in \mathbb{N}$ such that

(a3) $\lim _{m \rightarrow \infty} s_{n, m}(x, y)=1$ for $n \geq n_{0}$,

(b3) $\lim _{n \rightarrow \infty} s_{n, m}(x, y)=1$ for $m \geq m_{0}$,

(c3) $\lim _{n, m \rightarrow \infty} s_{n, m}(x, y)=1$.

If $(x, y)$ is a strict density point of a complement of $A$ then we say that $(x, y)$ is a strict dispersion point of $A$. Repeating the consideration related to strict density we obtain:

Proposition 3 A point $(x, y)$ is a strict density point of $A \in \mathcal{L}_{2}$ if and only if there is $k \in \mathbb{N}$ such that

(a4) $\lim _{m \rightarrow \infty} s_{k, m}(x, y)=1$;

(b4) $\lim _{n \rightarrow \infty} s_{n, k}(x, y)=1$;

(c4) $(x, y) \in \Phi_{s}(A)$.

Since we can choose a number $k$ independently for each point $(x, y)$, we have:

Proposition 4 If $(x, y)$ is an interior point of $A \in \mathcal{L}_{2}$ (in the natural topology on the plane), then $(x, y)$ is a strict density point of $A$.

Let denote by $\Phi_{s t}(A)$ the set of all strict density points of a measurable set $A$. For any $A \in \mathcal{L}_{2}$

$$
\Phi_{s t}(A) \subset \Phi_{s}(A)
$$

but those sets need not be equal. We shall show an example of set such that $\lambda_{2}\left(\Phi_{s}(A) \backslash\right.$ $\left.\Phi_{s t}(A)\right)>0$, but first we formulate a helpful proposition, similar to Theorem 1 .

Proposition 5 If, for any $h>0, \lambda_{1}([x-h ; x+h] \backslash E)>0$, then no point of the form $(x, y)$, where $y \in \mathbb{R}$, is a strict density point of a set $A=E \times \mathbb{R}$.

Example 3 Let $C \subset[0,1]$ be a nowhere dense, closed set of positive measure and $A=$ $C \times[0,1]$. By the last proposition, no point of $A$ is a strict density point of this set. Moreover, a set $\mathbb{R}^{2} \backslash A$ is open in the natural topology on the plane. Therefore, using Proposition 5 we 
obtain that $\Phi_{s t}(A)=\varnothing$. On the other hand, by the Lebesgue Density Theorem for the strong density ([4], Theorem 10.2), $\lambda_{2}\left(A \triangle \Phi_{s}(A)\right)=0$ and consequently

$$
\lambda_{2}\left(\Phi_{s}(A) \backslash \Phi_{s t}(A)\right)=\lambda_{1}(C)>0 .
$$

Theorem 4 For any $A \in \mathcal{L}_{2}$ a set $\Phi_{s t}(A)$ is measurable.

Proof Denote as usual

$$
s_{n, m}(x, y)=\frac{\lambda_{2}\left(A \cap\left(\left[x-\frac{1}{n} ; x+\frac{1}{n}\right] \times\left[y-\frac{1}{m} ; y+\frac{1}{m}\right]\right)\right)}{4 \cdot \frac{1}{n} \cdot \frac{1}{m}} .
$$

For any $k \in \mathbb{N}$ let

$$
A_{k}^{1}=\left\{(x, y) \in \mathbb{R}^{2}: s_{k, m}(x, y) \underset{m \rightarrow \infty}{\rightarrow} 1\right\}
$$

and

$$
A_{k}^{2}=\left\{(x, y) \in \mathbb{R}^{2}: s_{n, k}(x, y) \underset{n \rightarrow \infty}{\rightarrow} 1\right\} .
$$

From the definition of $\Phi_{s t}(A)$ we have

$$
\Phi_{s t}(A)=\Phi_{s}(A) \cap\left(\bigcup_{k \in \mathbb{N}}\left(A_{k}^{1} \cap A_{k}^{2}\right)\right) .
$$

Since $\lambda_{2}\left(A \triangle \Phi_{s}(A)\right)=0$, the set $\Phi_{s}(A)$ is measurable. We shall show that sets $A_{k}^{1}$ are also measurable. Observe that $(x, y) \in A_{k}^{1}$ if and only if

$$
\underset{p \in \mathbb{N}}{\forall} \underset{l \in \mathbb{N}}{\exists} \underset{m \geq l}{\forall} s_{k, m}(x, y) \geq 1-\frac{1}{p} .
$$

Denote by $A_{k, p, m}^{1}$ the set

$$
\left\{(x, y) \in \mathbb{R}^{2}: s_{k, m}(x, y) \geq 1-\frac{1}{p}\right\} .
$$

Therefore

$$
A_{k}^{1}=\bigcap_{p \in \mathbb{N}} \bigcup_{l \in \mathbb{N}}^{\infty} \bigcap_{m=l}^{1} A_{k, p, m} .
$$

Since $A_{k, p, m}^{1}$ is a closed set (because $s_{k, m}$ is continuous), $A_{k}^{1}$ is a $\mathcal{F}_{\sigma \delta}$ set, so it is measurable. In analogous way we show that, for any $k, A_{k}^{2}$ is a $\mathcal{F}_{\sigma \delta}$ set.

Remark 4 Since $A_{1}^{1}$ and $A_{2}^{1}$ are measurable and $\Phi_{r}(A)=\Phi_{s}(A) \cap\left(A_{k}^{1} \cap A_{k}^{2}\right)$, the operator $\Phi_{r}$ takes only measurable values.

Of course, no point of strict density of a set $A$ is a point of strict dispersion of this set. We shall show that if $\left(x_{0}, y_{0}\right)$ is a point of strict density of $A$, then no point of some cross centered at $\left(x_{0}, y_{0}\right)$ is a strong dispersion point of $A$. 
Theorem 5 If $\left(x_{0}, y_{0}\right)$ is a point of strict density of a measurable set $A$, then there exists $\delta>0$ such that no point of a set

$$
\left\{\left(x, y_{0}\right): x \in\left(x_{0}-\delta ; x_{0}+\delta\right)\right\} \cup\left\{\left(x_{0}, y\right): y \in\left(y_{0}-\delta ; y_{0}+\delta\right)\right\}
$$

is a strong dispersion point of $A$.

Proof Suppose that there is a sequence $\left\{x_{i}\right\}_{i \in \mathbb{N}}$ tending to $x_{0}$ such that $\left(x_{i}, y_{0}\right) \in \Phi_{s}\left(\mathbb{R}^{2} \backslash A\right)$ for $i \in \mathbb{N}$ and $\left(x_{0}, y_{0}\right) \in \Phi_{s t}(A)$. It follows that there is $n_{0} \in \mathbb{N}$ such that

$$
\lim _{m \rightarrow \infty} \frac{\lambda_{2}\left(\left(\mathbb{R}^{2} \backslash A\right) \cap\left(\left[x_{0}-\frac{1}{n_{0}} ; x_{0}+\frac{1}{n_{0}}\right] \times\left[y_{0}-\frac{1}{m} ; y_{0}+\frac{1}{m}\right]\right)\right)}{4 \cdot \frac{1}{n_{0}} \cdot \frac{1}{m}}=0 .
$$

Since $\lim _{i \rightarrow \infty} x_{i}=x_{0}$, there exists $i \in \mathbb{N}$ such that $x_{i} \in\left(x_{0}-\frac{1}{n_{0}} ; x_{0}+\frac{1}{n_{0}}\right)$. Using the assumption that $\left(x_{i}, y_{0}\right) \in \Phi_{s}\left(\mathbb{R}^{2} \backslash A\right)$ we can choose positive numbers $h_{0}$ and $k_{0}$ such that $\left(x_{i}-h_{0} ; x_{i}+h_{0}\right) \subset\left(x_{0}-\frac{1}{n_{0}} ; x_{0}+\frac{1}{n_{0}}\right)$ and for any $h \leq h_{0}$ and $k \leq k_{0}$

$$
\frac{\lambda_{2}\left(\left(\mathbb{R}^{2} \backslash A\right) \cap\left(\left[x_{i}-h ; x_{i}+h\right] \times\left[y_{0}-k ; y_{0}+k\right]\right)\right)}{4 h k} \geq \frac{1}{2} .
$$

For any $m \in \mathbb{N}$

$$
\begin{aligned}
& \left(\left[x_{i}-h_{0} ; x_{i}+h_{0}\right] \times\left[y_{0}-\frac{1}{m} ; y_{0}+\frac{1}{m}\right]\right) \\
& \quad \subset\left(\left[x_{0}-\frac{1}{n_{0}} ; x_{0}+\frac{1}{n_{0}}\right] \times\left[y_{0}-\frac{1}{m} ; y_{0}+\frac{1}{m}\right]\right) .
\end{aligned}
$$

Therefore, for $m>\frac{1}{k_{0}}$,

$$
\begin{aligned}
& \frac{\lambda_{2}\left(\left(\mathbb{R}^{2} \backslash A\right) \cap\left(\left[x_{0}-\frac{1}{n_{0}} ; x_{0}+\frac{1}{n_{0}}\right] \times\left[y_{0}-\frac{1}{m} ; y_{0}+\frac{1}{m}\right]\right)\right)}{4 \cdot \frac{1}{n_{0}} \cdot \frac{1}{m}} \\
& \geq \frac{\frac{1}{2} 4 h_{0} \frac{1}{m}}{\frac{4}{n_{0} m}}=\frac{n_{0} h_{0}}{2} .
\end{aligned}
$$

It contradicts the condition (\#) and ends the proof that no point from the interval $\left\{\left(x, y_{0}\right)\right.$ : $\left.x \in\left(x_{0}-\delta ; x_{0}+\delta\right)\right\}$ is a strong dispersion point of $A$. The rest of the proof runs in the same manner.

Similarly to the properties of operator $\Phi_{r}$ one can prove

Proposition 6 The operator $\Phi_{s t}: \mathcal{L}_{2} \rightarrow 2^{\mathbb{R}^{2}}$ has the following properties:

(1) for each $A, B \in \mathcal{L}_{2}$, if $\lambda_{2}(A \triangle B)=0$, then $\Phi_{s t}(A)=\Phi_{s t}(B)$;

(2) $\Phi_{s t}(\varnothing)=\varnothing$ and $\Phi_{s t}\left(\mathbb{R}^{2}\right)=\mathbb{R}^{2}$;

(3) for each $A, B \in \mathcal{L}_{2}, \Phi_{s t}(A \cap B)=\Phi_{s t}(A) \cap \Phi_{s t}(B)$.

Straightforward from (3) it follows that an operator $\Phi_{s t}$ is monotonic i.e. for each $A$, $B \in \mathcal{L}_{2}$ if $A \subset B$, then $\Phi_{s t}(A) \subset \Phi_{s t}(B)$. Moreover 
Theorem 6 A family

$$
\mathcal{T}_{s t}=\left\{A \subset \mathbb{R}^{2}: A \in \mathcal{L}_{2} \text { and } A \subset \Phi_{s t}(A)\right\}
$$

is a topology on the plane stronger than the natural topology and weaker than the strong density topology.

Proof It is clear that $\varnothing \in \mathcal{T}_{s t}$ and $\mathbb{R}^{2} \in \mathcal{T}_{s t}$. By virtue of the condition (3) from the previous proposition, a family $\mathcal{T}_{s t}$ is closed under finite intersection. Suppose that $A_{\gamma} \in \mathcal{T}_{s t}$ for any $\gamma \in \Gamma$. Then $\left(\bigcup_{\gamma \in \Gamma} A_{\gamma}\right) \subset \Phi_{s t}\left(\bigcup_{\gamma \in \Gamma} A_{\gamma}\right)$ because $\Phi_{s t}$ is monotone. To show that $\cup_{\gamma \in \Gamma} A_{\gamma}$ is measurable we shall use an inclusion $\Phi_{s t}(A) \subset \Phi_{s}(A)$ for any $A \in \mathcal{L}_{2}$ and the fact that a family

$$
\mathcal{T}_{s}=\left\{A \subset \mathbb{R}^{2}: A \in \mathcal{L}_{2} \text { and } A \subset \Phi_{s}(A)\right\}
$$

forms a topology, called a strong density topology (it is proved in [1], using the Vitali covering theorem). In particular, it follows that a set $\bigcup_{\gamma \in \Gamma} A_{\gamma}$, as a union of sets from $\mathcal{T}_{s}$, is measurable.

Denote by $\mathcal{T}_{o}$ the natural topology on the plane. From definitions and Proposition 4 we obtain

$$
\mathcal{T}_{o} \subset \mathcal{T}_{s t} \subset \mathcal{T}_{s}
$$

Let $A=C \times[0 ; 1]$ be the set constructed in Example 3 and $B=A \cap \Phi_{s}(A)$. Since $\lambda_{2}(A \triangle$ $\left.\Phi_{s}(A)\right)=0$, we know that $\lambda_{2}(B)>0$ and $\Phi_{s}(B)=\Phi_{s}(A) \supset B$. Therefore, $B \in \mathcal{T}_{s}$. On the other hand,

$$
\Phi_{s t}(B) \subset \Phi_{s t}(A)=\varnothing
$$

so $B \notin \mathcal{T}_{\text {st }}$.

To construct a set belonging to $\mathcal{T}_{s t} \backslash \mathcal{T}_{o}$ we use the set $A$ constructed in Example 1. Let

$$
D=\mathbb{R}^{2} \backslash A \cup\{(0,0)\} .
$$

It is easily seen that a point $(0,0)$ is not an interior point of $D$ in the natural topology and, consequently, $D \notin \mathcal{T}_{o}$. However, $(0,0)$ is a restricted density point of $D$ and its strict density point. The other points of $D$ belong to $\Phi_{s t}(B)$ because $D \backslash\{(0,0)\} \in \mathcal{T}_{o}$. Therefore $D \in \mathcal{T}_{\text {st }}$.

Theorem 7 The topology $\mathcal{T}_{\text {st }}$ is stronger then the Hashimoto topology $\mathcal{T}_{H}$ defined by the formula

$$
\mathcal{T}_{H}=\left\{U \backslash P: U \in \mathcal{T}_{o}, \lambda_{2}(P)=0\right\}
$$

Proof For any set of the form $U \backslash P$, where $U \in \mathcal{T}_{o}$ and $\lambda_{2}(P)=0$, by virtue of Proposition 4 and the condition (1) from Proposition 6, we have

$$
\Phi_{s t}(U \backslash P)=\Phi_{s t}(U)=U .
$$

Therefore $U \backslash P \in \mathcal{T}_{s t}$. As an example of set belonging to $\mathcal{T}_{s t} \backslash \mathcal{T}_{H}$ we can use the set $D$ considered in the previous proof.

Theorem 8 A topological space $\left(\mathbb{R}^{2}, \mathcal{T}_{\text {st }}\right)$ is Hausdorff but not regular. 
Proof A space $\left(\mathbb{R}^{2}, \mathcal{T}_{s t}\right)$ is a Hausdorff space, because $\mathcal{T}_{o} \subset \mathcal{T}_{s t}$. To prove that it is not regular, observe first that any set of measure zero is closed in $\left(\mathbb{R}^{2}, \mathcal{T}_{s t}\right)$. Therefore the set

$$
P=\{(x, 0): x \neq 0\}
$$

is closed in $\left(\mathbb{R}^{2}, \mathcal{T}_{s t}\right)$. By virtue of Theorem 4 there is impossible to find disjoint sets $G_{1}$ and $G_{2}$, belonging to $\mathcal{T}_{s t}$, such that $(0,0) \in G_{1}$ and $P \subset G_{2}$.

Acknowledgement $\quad$ W. Wilczynski was supported by grant number: 5472/B/H03/2010/38 .

Open Access This article is distributed under the terms of the Creative Commons Attribution Noncommercial License which permits any noncommercial use, distribution, and reproduction in any medium, provided the original author(s) and source are credited.

\section{References}

1. Goffman, C., Neugebauer, C.J., Nishiura, T.: The density topology and approximate continuity. Duke Math. J. 28, 497-506 (1961)

2. Filipczak, M., Wilczyński, W.: Remarks on area density. Reports on Real Analysis, pp. 37-49, Słupsk (2001)

3. Oxtoby, J.C.: Measure and Category. Springer, Berlin (1971)

4. Saks, S.: Theory of the integral, Warszawa-Lwów (1937)

5. Tschelidze, W.S.: Methods of summability of double sequences and double integrals, University of Tbilisi (1977) (in Russian) 